\title{
ÁCIDOS ORGÂNICOS DE BAIXO PESO MOLECULAR E ÁCIDOS HÚMICOS E ALTERAÇÕES EM ALGUMAS PROPRIEDADES FÍSICAS E QUÍMICAS DE LATOSSOLOS, PLINTOSSOLO E NEOSSOLO QUARTZARÊNICO ${ }^{(1)}$
}

\author{
Marcelo Metri Corrêa ${ }^{(2)}$, Felipe Vaz de Andrade ${ }^{(3)}$, Eduardo Sá \\ Mendonça $^{(4)}$, Carlos Ernesto G.R. Schaefer ${ }^{(4)}$, Tiago Torres Costa \\ Pereira $^{(5)}$ \& Cecília Calhau Almeida ${ }^{(6)}$
}

\section{RESUMO}

Os ácidos orgânicos provenientes da decomposição da matéria orgânica, da exsudação radicular e do metabolismo de microrganismos, possuem importante papel na melhoria das condições físicas e químicas do solo. Entretanto, sua eficiência está relacionada à qualidade e à forma dos ácidos orgânicos e à sua interação com colóides do solo. Com o objetivo de avaliar o efeito dos ácidos orgânicos de alto e baixo peso molecular na alteração de propriedades físicas e químicas de solos, foram coletados materiais de horizontes B de quatro Latossolos e um Plintossolo, e C de Neossolo Quartzarênico. Para isso, foram utilizadas doses de ácidos cítricos e oxálicos de $0,1,3,9$ e $18 \mathrm{mmol} \mathrm{L}^{-1}$; e para os ácidos húmicos, doses de 0,$0 ; 2,0 ; 4,0 ; 6,0$ e 10,0 $\mathrm{g} \mathrm{kg}^{-1}$, num delineamento experimental inteiramente casualizado seguindo um esquema fatorial de $6 \times 3 \times 5$ (solo, ácido e dose), com três repetições. As doses utilizadas foram de $0,1,3,9$ e $18 \mathrm{mmol} \mathrm{L}^{-1}$ para os ácidos cítrico e oxálico e, para os ácidos húmicos, de 0,$0 ; 2,0 ; 4,0 ; 6,0 ;$ e $10,0 \mathrm{~g} \mathrm{~kg}^{-1}$. As unidades experimentais foram compostas de $25 \mathrm{~cm}^{3}$ de TFSA, colocadas em cilindros de PVC (2,0 cm de altura por $4,0 \mathrm{~cm}$ de diâmetro). Estas foram mantidas em câmaras isotérmicas a $30^{\circ} \mathrm{C}$ e submetidas a ciclos de umedecimento e secagem de três dias, por sete ciclos. Ao final do experimento foram determinados o teor de argila dispersa em água, a resistência à penetração e os teores de Fe e $\mathrm{Al}$ por oxalato ácido de amônio. Os resultados mostraram que o conteúdo de argila dispersa

\footnotetext{
(1) Recebido para publicação em janeiro de 2007 e aprovado em outubro de 2007.

(2) Professor Adjunto da Unidade Acadêmica de Garanhuns, Universidade Federal Rural de Pernambuco - UFRPE. CEP 552986280 Garanhuns (PE). E-mail: marcelometri@yahoo.com

(3) Professor Adjunto do Departamento de Produção Vegetal - Universidade Federal do Espírito Santo - CCA/UFES. Alto Universitário s/n, Caixa Postal 16, Centro, CEP 29500-000 Alegre (ES). E mail: fvandrade@cca.ufes.br

(4) Professor do Departamento de Solos, Universidade Federal de Viçosa - UFV. Av. PH Rolfs sn, CEP 36570-000 Viçosa (MG). E-mails: esm@ufv.br; carlos.schaefer@ufv.br

(5) Mestrando do Departamento de Solos, UFV. E-mail: torresthiago@ yahoo.com.br

${ }^{(6)}$ Mestranda do Departamento de Solos, UFV. E-mail: ceciliaufv@yahoo.com.br
} 


\begin{abstract}
variou com o tipo e dose dos ácidos orgânicos, textura e mineralogia dos solos, indicando que aqueles goethíticos apresentaram maior resistência à dispersão que os hematíticos, e estes, por sua vez, maior resistência que os gibbsíticos. Com relação aos ácidos, foi observado que os de cadeia curta promoveram maior dispersão e resistência à penetração que os ácidos de cadeia longa, podendo-se estabelecer a seguinte ordem, de acordo com seu efeito: ácido cítrico $\geq$ ácido oxálico $>$ ácidos húmicos. $O$ conteúdo de argila dispersa em água mostrou ser o principal fator responsável pelo aumento da resistência à penetração.
\end{abstract}

Termos de indexação: ácido cítrico, ácido oxálico, ácido húmico.

\title{
SUMMARY: LOW MOLECULAR WEIGHT ORGANIC AND HUMIC ACIDS AND EFFECTS ON PHYSICAL AND CHEMICAL PROPERTIES OF OXISOLS AND RELATED SOILS
}

\begin{abstract}
The organic acids from the organic matter decomposition, root exudation and microbial activity, play an important role on physical and chemical soil conditions. However their efficiency is conditioned to the quality and kind of organic acids and interactions with the soil mineral surface. The aim of this study was to evaluate the effect of humic and organic acids of high and low molecular weight on the physical and chemical properties of $B$ horizons of four Oxisols and one Plinthustult, and a $C$ horizon of Quartzipsamment. A completely randomized $6 \times 3 \times 5$ factorial design (soils $\mathrm{x}$ organic acids $\mathrm{x}$ dose) was used, with 3 replications. The following organic acid rates were applied: $0,1,3,9$ and $18 \mathrm{mM}$ citric and oxalic acid and $0.0 ; 2.0 ; 4.0 ; 6.0$ and $10.0 \mathrm{~g} \mathrm{~kg}^{-1}$ humic acids. The experimental units consisted of $25 \mathrm{~cm}^{3}$ of air-dried fine-earth fraction in PVC cylinders (2.0 cm height and $4.0 \mathrm{~cm}$ diameter), subjected to moistening and drying cycles in an isothermal chamber at $30{ }^{\circ} \mathrm{C}$, for three days, in seven cycles. The water dispersible clay, penetration resistance and ammonium oxalate-extracted $F e$ and $A l$ were determined. The contents of water dispersible clay were variable, related to the rate and type of organic acids, and also to soil texture and mineralogy. Thus, the dispersion resistance of the goethitic was higher than of the hematitic soils, which was in turn higher than of the gibbsitic soils. Moreover, organic acids of low molecular weight resulted in higher dispersion and penetration resistance than those of high molecular weight, in the following sequence: citric acid ${ }^{3}$ oxalic acid > humic acid. The content of water dispersible clay proved to be an important factor in the increase of penetration resistance.
\end{abstract}

Index terms: citric acid, humic acid, oxalic acid, Oxisols.

\section{INTRODUÇÃO}

A floculação e dispersão de minerais de argila têm recebido, ao longo dos anos, grande atenção dos pesquisadores, dada sua importância em processos industriais e pedogenéticos. Nestes últimos, destacase o fenômeno de formação e estabilização dos agregados dos solos, explicado a partir de várias hipóteses que têm como componente principal da matéria orgânica, principalmente as substâncias húmicas, como importante agente de ligação dos constituintes minerais dos solos (Emerson, 1959; Edwards \& Bremner, 1967; Tisdall \& Oades, 1982). Paradoxalmente, contudo, várias substâncias orgânicas podem agir como dispersantes em sistemas multifases. Como defendem alguns autores (Durgin \& Chaney, 1984; Visser \& Caillier, 1988; Benites \& Mendonça, 1998; Tombácz et al., 2004), os materiais orgânicos podem levar tanto à floculação como à dispersão; o predomínio de forças repulsivas ou atrativas vai depender da qualidade e quantidade de partículas minerais e complexantes orgânicos.
Dentre as várias características dos constituintes coloidais do solo, as de superfície (cargas e área específica) mostram grande influência na formação e estabilização de agregados. Dessa forma, todos os fatores que alteram a interface elétrica entre os constituintes do solo podem interferir no processo de floculação e dispersão. Entre esses fatores encontrase a adsorção de ânions orgânicos de alto (fúlvico e húmico) (Tarchitzky et al., 1993; Tombácz et al., 2004) ou baixo peso molecular (ácidos oxálico, cítrico, málico, etc.) (Shanmuganathan \& Oades, 1983; Xu et al., 2004).

A adsorção desses constituintes orgânicos pode ocorrer de diferentes modos, intensidades e teor, dependendo das características do solo, como: constituição mineralógica, $\mathrm{pH}$ e força iônica da solução (Tomácz et al., 2004). Além dos fatores edáficos, características dos ânions orgânicos, como o tamanho da cadeia de C, a disposição dos grupamentos funcionais e a densidade de carga, interferem na adsorção com os constituintes do solo. 
A interação ânion orgânico com os óxidos de Fe e $\mathrm{Al}$ e com minerais de argila 1:1 pode se dar por meio de força de Coulomb (adsorção não-específica) ou, predominantemente, por coordenação (Greenland, 1971), denominado por Hingston et al. (1967) de ligação específica. Esta pode envolver troca de ligante entre os grupamentos $\mathrm{OH}$ dos radicais orgânicos (carboxílicos e fenólicos) e Fe-OH e Al-OH dos colóides inorgânicos.

Tambácz et al. (2004) afirmam que a quantidade adsorvida de polímeros orgânicos pela hematita e pela magnetita foi superior àquela adsorvida por minerais de argila aluminossilicatados (montmorilonita e caulinita). Em geral, os componentes orgânicos agem alterando as cargas positivas para negativas dos sítios ativos dos minerais de argilas e óxidos (Al-OH dos óxidos de $\mathrm{Al}$ e das bordas quebradas dos minerais de argilas e $\mathrm{Fe}-\mathrm{OH}$ dos óxidos de $\mathrm{Fe}$ ). Esse processo geraria novo PI (ponto isoelétrico) para os colóides inorgânicos e, dependendo do $\mathrm{pH}$ do sistema, conduziria à repulsão ou à atração das partículas (Tombácz et al., 2004).

A dispersão dos constituintes do solo pode conduzir ao colapso estrutural, com conseqüente favorecimento da erosão superficial, formação de camadas adensadas, iluviação de argilas e complexos organometálicos (podzolização), etc. Shanmuganathan \& Oades (1983), estudando a influência de ácidos orgânicos de baixo peso molecular nas propriedades físicas de solos vermelhos da Austrália, concluíram que a formação de argila dispersa pelos tratamentos conduziu ao aumento da densidade do solo e dos limites de plasticidade e de ruptura, e diminuição da porosidade e condutividade hidráulica.

Diante do exposto, o presente trabalho teve como objetivo analisar a relação entre os ácidos húmicos, cítrico e oxálico e a geração de propriedades físicas indesejáveis em solos tropicais brasileiros com características texturais e mineralógicas distintas.

\section{MATERIAL E MÉTODOS}

\section{Delineamento experimental}

Foram coletadas seis amostras de horizonte B de solos de diferentes classes, regiões, texturas e domínio mineralógico (Quadro 1). As amostras foram secas ao ar, destorroadas e passadas em peneira de malha de $2 \mathrm{~mm}$, para obtenção da TFSA.

Adicionaram-se às amostras diferentes doses de ácidos cítrico $(\mathrm{AC})$, oxálico $(\mathrm{AO})$ e húmicos $(\mathrm{AH})$. O delineamento experimental utilizado foi o inteiramente casualizado, em esquema fatorial 6 × 3 x 5 (solo, ácido e dose), com três repetições. As doses utilizadas foram de $0,1,3,9$ e $18 \mathrm{mmol} \mathrm{L}^{-1}$ para os ácidos cítrico e oxálico e, para os ácidos húmicos, de 0,$0 ; 2,0 ; 4,0 ; 6,0$; e 10,0 $\mathrm{g} \mathrm{kg}^{-1}$ de solo. As soluções dos ácidos orgânicos foram ajustadas a $\mathrm{pH} 5$ e aplicadas por ascensão capilar, de maneira que a dose aplicada atingisse a capacidade de campo para cada amostra de solo estudado.

Para $\mathrm{AC}$ e $\mathrm{AO}$ foi utilizado reagente $\mathrm{PA}$. Os ácidos húmicos foram extraídos de um Espodossolo da região de Lavras Novas (Minas Gerais, Brasil), purificados e dialisados, conforme as normas e orientações da Sociedade Internacional de Substâncias Húmicas IHSS (Swift, 1996).

Amostras de $25 \mathrm{~cm}^{3}$ de TFSA foram colocadas em cilindros de PVC $(2,0 \mathrm{~cm}$ de altura por $4,0 \mathrm{~cm}$ de diâmetro), em que se considerou a densidade do solo

\section{Quadro 1. Características físicas e químicas de amostras dos solos estudados}

\begin{tabular}{|c|c|c|c|c|c|c|c|c|}
\hline Solo & Classe & Região & Material de origem & $F e_{d}^{(1)}$ & $\mathrm{Fe}_{\mathrm{o}}^{(1)}$ & $\mathrm{Fe}_{\mathrm{o}} / \mathrm{Fe}_{\mathrm{d}}$ & $\operatorname{Argila}^{(2)}$ & $\begin{array}{c}\text { Domínio } \\
\text { mineralógico } \\
\text { (argila) }\end{array}$ \\
\hline & & & & \multicolumn{2}{|c|}{$-\mathrm{mg} \mathrm{g}^{-1}-$} & \multicolumn{2}{|r|}{$\mathrm{g} \mathrm{kg}^{-1}$} & \\
\hline RQ & $\begin{array}{l}\text { Neossolo } \\
\text { Quartzarênico }\end{array}$ & Ilhéus (BA) & Sedimentos arenosos & - & - & - & 90 & Caulinita \\
\hline $\mathrm{FX}$ & Plintossolo Háplico & Romaria (MG) & $\begin{array}{l}\text { Material retrabalhado } \\
\text { sobre basalto }\end{array}$ & 2,51 & 0,32 & 0,13 & 680 & Gibbsita e caulinita \\
\hline LVA1 & $\begin{array}{l}\text { Latossolo } \\
\text { Vermelho-Amarelo }\end{array}$ & João Pinheiro (MG) & $\begin{array}{l}\text { Sedimento argilo- } \\
\text { arenoso }\end{array}$ & 55,3 & 0,50 & 0,01 & 300 & Caulinita e goethita \\
\hline LVA2 & $\begin{array}{l}\text { Latosssolo } \\
\text { Vermelho-Amarelo }\end{array}$ & Viçosa (MG) & Gnaisse & 54,73 & 0,62 & 0,01 & 670 & Caulinita e goethita \\
\hline LV1 & Latossolo Vermelho & Igarapava (SP) & Basalto e arenito & 43,31 & 1,36 & 0,03 & 280 & Caulinita e hematita \\
\hline LV2 & $\begin{array}{l}\text { Latossolo } \\
\text { Vermelho acriférrico }\end{array}$ & Abre Campo (MG) & $\begin{array}{l}\text { Gnaisse c/ influência } \\
\text { máfica }\end{array}$ & 87,15 & 1,88 & 0,02 & 700 & Caulinita e hematita \\
\hline
\end{tabular}

(1) Determinado segundo procedimento descrito em MacKeague (1978). ${ }^{(2)}$ Determinado segundo procedimento descrito em Embrapa (1997). 
por classe textural (argilosas, $\mathrm{Ds}=0,90 \mathrm{~g} \mathrm{~cm}^{-3}$; média e arenosas, Ds $\left.=1,10 \mathrm{~g} \mathrm{~cm}^{-3}\right)$. As amostras foram mantidas em câmaras isotérmicas a $30{ }^{\circ} \mathrm{C}$ e submetidas a sete ciclos de umedecimento e secagem de três dias, ou seja, reumedecidas à capacidade de campo por meio de novas aplicações das soluções dos ácidos orgânicos de três em três dias. Especificamente para os ácidos húmicos, a aplicação foi realizada de tal forma que, no final dos sete ciclos, a soma da quantidade aplicada correspondesse às doses anteriormente descritas.

Os efeitos da mineralogia e teor de argila dos solos na resistência máxima à penetração, da argila dispersa em água e dos teores de Fe e Al extraídos por oxalato foram estudados por contrastes (Quadro 2). Os efeitos do aumento das doses foram analisados por regressão linear.

\section{Argila dispersa em água e resistência à penetração}

Os materiais de solo foram removidos dos anéis e, novamente, destorroados e passados em peneiras de malha de $2 \mathrm{~mm}$. Para determinação da argila dispersa em água, utilizou-se o método da pipeta (Embrapa, 1997). Neste, foi deixada em repouso por 6 h uma suspensão composta de 2,5 g de solo e $30 \mathrm{~mL}$ de água deionizada. Em seguida, essas amostras foram transferidas para tubos de centrífuga e agitadas por $16 \mathrm{~h}$ em agitador vertical. A determinação foi feita em provetas de $250 \mathrm{~mL}$.

\section{Quadro 2. Contrastes considerados}

\begin{tabular}{|c|c|c|c|c|c|}
\hline \multirow{2}{*}{ Composto orgânico } & \multicolumn{5}{|c|}{ Contraste } \\
\hline & \multicolumn{2}{|r|}{1} & \multicolumn{3}{|c|}{2} \\
\hline $\mathrm{AC}$ & & + & & + & \\
\hline $\mathrm{AO}$ & & + & & . & \\
\hline $\mathrm{AH}$ & & - & & c & \\
\hline \multirow{2}{*}{ Solo } & \multicolumn{5}{|c|}{ Contraste } \\
\hline & 3 & 4 & 5 & 6 & 7 \\
\hline $\mathrm{LV}_{1}$ & $-1,5$ & + & 0 & 0 & + \\
\hline $\mathrm{LV}_{2}$ & + & 0 & + & 0 & 0 \\
\hline $\mathrm{LVA}_{1}$ & $-1,5$ & + & 0 & 0 & - \\
\hline $\mathrm{LVA}_{2}$ & + & 0 & - & - & 0 \\
\hline $\mathrm{FX}$ & + & 0 & 0 & + & 0 \\
\hline $\mathrm{RQ}$ & 0 & -2 & 0 & 0 & 0 \\
\hline
\end{tabular}

AC: ácido cítrico; $\mathrm{AO}$ : ácido oxálico; $\mathrm{AH}$ : ácido húmico; $\mathrm{AC}+\mathrm{AO}$ : combinação ácido cítrico e ácido oxálico; $\mathrm{C}_{1}=\mathrm{AC}+\mathrm{AO}$ vs $\mathrm{AH}$; $\mathrm{C}_{2}=\mathrm{AC}$ vs $\mathrm{AO} ; \mathrm{C}_{3}=\mathrm{LV}_{2}+\mathrm{LVA}_{2}+\mathrm{FX} v \mathrm{LVA} \mathrm{LV}_{1}+\mathrm{LV}_{1} ; \mathrm{C}_{4}=\mathrm{LV}_{1}+$ $\mathrm{LVA}_{1}$ vs RQ; $\mathrm{C}_{5}=\mathrm{LV}_{2}$ vs LVA $\mathrm{LC}_{6}=\mathrm{FX}$ vs $\mathrm{LVA}_{2} ; \mathrm{C}_{7}=\mathrm{LV}_{1}$ vs $\mathrm{LVA}_{1}$.
A resistência à penetração foi determinada em aparelho Marconi, modelo MA-933, utilizando cone de $4 \mathrm{~mm}$ de diâmetro, $60^{\circ}$ de inclinação (ponteira) e avanço de $10 \mathrm{~mm} \mathrm{~min}^{-1}$ e leitura a cada $1 \mathrm{~s}$. As medidas foram tomadas no centro das amostras (cilindros) previamente secas ao ar. De acordo com Hansbo (1957), a relação entre a força de corte (T) e a força experimental (Q) é dada pela equação:

$$
T=K \frac{Q}{h^{2}}\left(\frac{k g}{m^{2}}\right)
$$

em que $\mathrm{K}$ é um coeficiente que depende, predominantemente, do cone de penetração e, parcialmente, do material do solo, e h, a profundidade de penetração $(\mathrm{m})$. Como o K é desconhecido para os solos estudados e foi utilizado o mesmo cone em todas as leituras, pode-se considerar a relação $\mathrm{Q} / \mathrm{h}^{2}$ como a propriedade denominada resistência à penetração. Foi utilizado nas análises estatísticas o valor máximo observado $\left(\mathrm{R}_{\max }\right)$. No presente trabalho, os valores de $\mathrm{R}_{\max }$ foram convertidos em $\mathrm{kPa}$, utilizando-se o fator 98,07 .

\section{Fe e Al extraídos por oxalato}

Os elementos $\mathrm{Al}$ e $\mathrm{Fe}$ foram determinados na fração TFSA por espectroscopia de emissão de plasma, após uma única extração com oxalato ácido de amônio, segundo método constante em MacKeague (1978).

\section{Mineralogia}

A fração argila foi separada e seus componentes foram identificados por difratometria de raios $\mathrm{X}$. Na determinação dos valores da componente de GaussCauchy (n), da largura à meia altura (LMC) e da dimensão média do cristal no plano 001 (DMC001) foi utilizado o software Rietica (Hunter \& Howard, 2000) e a função de ajuste de pico pseudo-Voigt, ajustandose vários parâmetros, que incluem os da célula unitária, zero $2 \theta$, fator de escala e outros. A LMC do pico foi modelada a partir de uma função quadrática com três parâmetros refináveis, representados por U, V e W (Caglioti et al., 1958). Quando necessário, os parâmetros da célula unitária foram refinados, seletivamente. A linha de base foi modelada por uma função polinomial com quatro parâmetros. Para descrever a qualidade do refinamento, foram observados os índices $\mathrm{R}$ e a linha de diferença, que representa o desvio entre a difração observada e a calculada.

\section{RESULTADOS E DISCUSSÃO}

\section{Mineralogia}

Por meio dos espectros de DRX (Figura 1), observou-se ampla variação na mineralogia dos solos estudados. No geral, a fração argila apresentou 
caulinita (Ct) e gibbsita (Gb) como minerais comuns para todos os solos estudados. Os outros minerais identificados foram: hematita $(\mathrm{Hm})$, goethita $(\mathrm{Gt})$, anatásio (An) e zircão (Zi); este último foi apenas identificado no Plintossolo de Romaria - MG (FX).

A presença marcante de Gb no FX está relacionada, possivelmente, ao baixo teor de Si do material de origem (materiais retrabalhados sobre basalto) e à condição de hidromorfismo em que se encontra o referido solo. Nesse ambiente, a presença de ciclos redox de $\mathrm{Fe}$ conduz a uma constante produção de $\mathrm{H}^{+}$, que, por sua vez, acidificam o meio e promovem a dissolução de caulinita (acidólise), liberando Si e Al para o meio. O Si é removido do sistema por lixiviação e o $\mathrm{Al}$, menos móvel, precipita na forma de gibbsita. Os minerais An e Zi também se mostraram expressivos nesse solo, em razão da sua alta resistência às reações propostas. $\mathrm{A} \mathrm{Gb}$ também foi identificada nos solos $\mathrm{LV}_{1}$ e $\mathrm{LV}_{2}$, em razão do baixo teor de Si de seus materiais de origem (influência de materiais alcalinos). Do mesmo modo, nestes solos também foi identificada a presença de $\mathrm{Hm}$. A maguemita foi identificada no $\mathrm{LV}_{1}$ (originado de basalto) tanto por DRX como por atração magnética, por meio de ímã de mão em laboratório.

No quadro 3 estão descritos os valores da componente de Gauss-Cauchy (n) e valores de LMA e DMC do pico 001 da caulinita da argila dispersa na dose de $3 \mathrm{mmol} \mathrm{L}^{-1}$ de ácido cítrico e na testemunha. Todos foram obtidos utilizando-se a função de ajuste de forma de pico pseudo-Voigt. Os valores de DMC variaram de 10 a $38 \mathrm{~nm}$, estando de acordo com Ker (1995).

Além do DMC, os valores de n e LMA também variaram com o solo estudado. Para os solos $\mathrm{LV}_{2}$, $\mathrm{LVA}_{2}$ e FX, foi observada variação no DMC entre a testemunha e a dose de $3 \mathrm{mmol} \mathrm{L}^{-1}$ de ácido cítrico, sugerindo maior na dispersão das partículas de menor tamanho. Esse fato pode ser decorrente de sua maior superfície específica por grama de material. A não observação dessa variação nos demais solos deve-se, possivelmente, à maior homogeneidade no tamanho da sua fração argila, com domínio marcante de partícula de pequeno $\mathrm{DMC}_{001}$.

\section{Argila dispersa em água}

Os resultados demonstraram que os ácidos estudados interferiram de forma marcante e diferenciada no processo de dispersão e floculação dos colóides inorgânicos dos solos (Quadro 4). Os valores de $\mathrm{AD}$ e $\mathrm{R}_{\max }$ permitiram separar os ácidos em dois grupos, de acordo com seu efeito: ácidos cítrico e oxálico (baixo peso molecular) e ácidos húmicos (alto peso molecular). Pelo contraste $\mathrm{C}_{1}$ (Quadro 4), observaramse valores superiores de $\mathrm{AD}$ e $\mathrm{R}_{\max }$ para os ácidos de baixo peso molecular. Esse fato deve-se à maior interação desses ânions com as partículas coloidais inorgânicas dos solos, que se dá de forma mais específica (troca de ligantes), alterando o potencial isoelétrico (PI) dos minerais de argilas e óxidos.

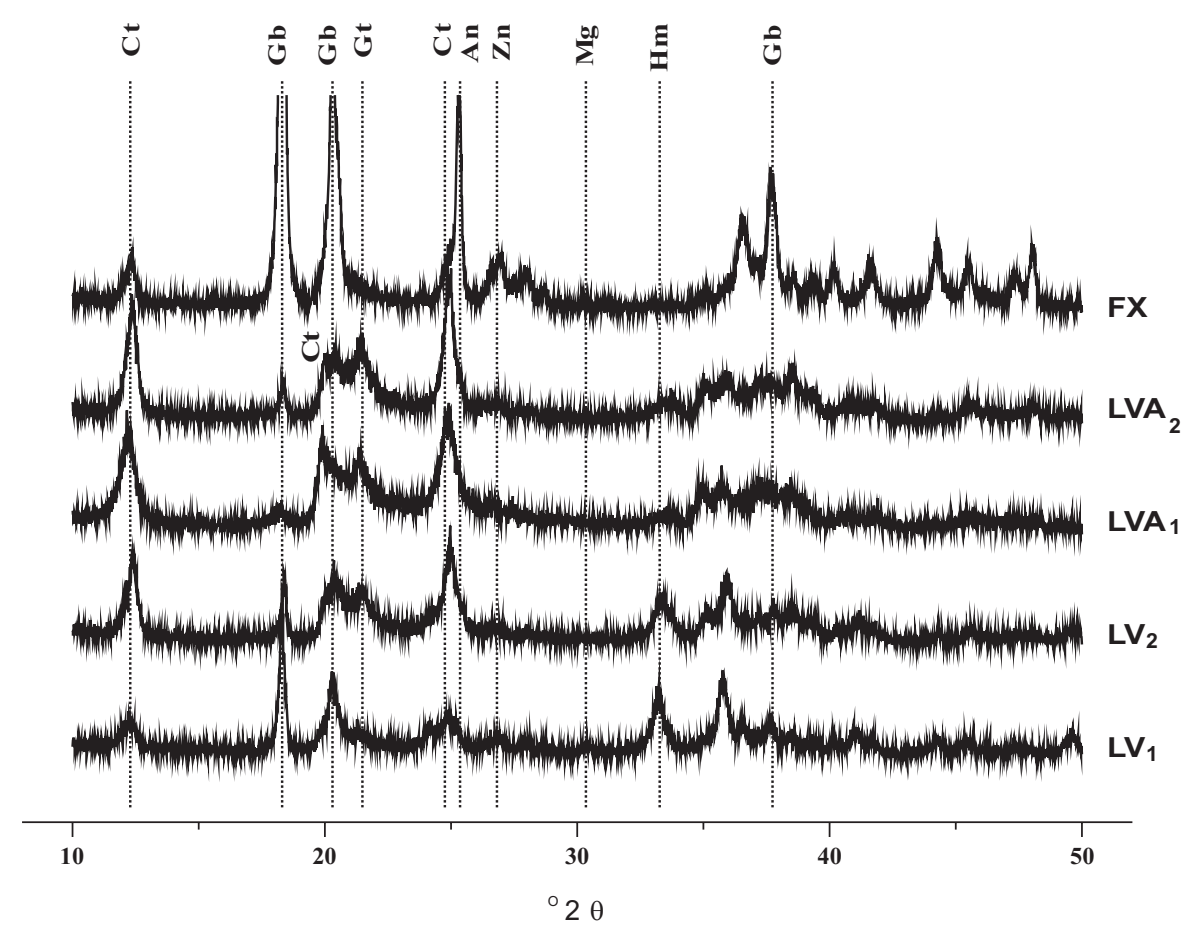

Figura 1. Espectros de difratometria de raios $X(\mathrm{Cu} k \alpha)$ da fração argila natural dos solos de textura média e argilosa estudados. Ct: caulinita; Gb: gibbsita; Gt: goethita; Hm: hematita; Mg: maguemita; An: anatásio; Zn: zircão. Os demais reflexos não identificados são picos dos minerais anteriormente descritos. 
Essa ligação neutraliza cargas positivas, gerando excesso de cargas negativas (Tombácz et al., 2004), ocasionando forte repulsão e, conseqüentemente, dispersão das partículas do solo. Durgin \& Chaney (1984) ressaltam que, além de bloquear cargas positivas, os polímeros orgânicos podem gerar cargas negativas em razão dos seus grupos funcionais, principalmente carboxílicos e fenólicos.

Embora não ocorrendo diferença significativa entre os efeitos dos ácidos cítrico e oxálico na $\mathrm{AD}$ (contraste $\mathrm{C}_{2}$ ), observou-se tendência de maiores valores para os tratamentos com ácido cítrico (Figura 2). No $\mathrm{LVA}_{1} \mathrm{e}$ $\mathrm{LVA}_{2}$ essa tendência não é mostrada. De acordo com alguns autores (Shanmuganathan \& Oades, 1983; Durgin \& Chaney, 1984; Andrade, 2001), isso se dá por causa do maior número de grupamentos funcionais (carboxílicos e alcoólico) e da maior especificidade na adsorção. Andrade (2001) relata ainda a possibilidade de a disposição dos grupamentos influenciar a dispersão de colóides. $\mathrm{O}$ grupamento $\mathrm{OH}$, entre grupamentos carboxílicos, conferiria um múltiplo ponto de contato, dando um caráter de entropia à adsorção. Dessa forma, sugere-se a seguinte ordem decrescente para o efeito dos ânions orgânicos na dispersão: ácido cítrico, ácido oxálico e ácido húmico. Resultados semelhantes foram encontrados por Shanmuganathan \& Oades (1983).

Para o ácido húmico (Figura 3), percebeu-se grande variação de seu efeito na dispersão para os solos estudados, ora diminuindo o teor de argila dispersa (FX e $\left.\mathrm{LV}_{2}\right)$, ora aumentando $\left(\mathrm{LV}_{1}, \mathrm{LVA}_{1}\right.$ e RQ), ou, ainda, sem interferência na dispersão $\left(\mathrm{LVA}_{2}\right)$. Os materiais orgânicos podem conduzir tanto à floculação como à dispersão; o predomínio de forças repulsivas

Quadro 3. Valores da componente de Gauss-Cauchy (n), largura à meia altura (LMA) e diâmetro médio do cristalito (DMC) da fração argila natural (testemunha) e dispersa na dose de ácido cítrico de $3 \mathrm{mmol} \mathrm{L}^{-1}$ $\left(\mathrm{C}_{3}\right)$ dos solos de textura média e argilosa estudados

\begin{tabular}{|c|c|c|c|c|}
\hline Amostra & Concentração & $\mathbf{n}$ & LMA & $\mathbf{D} \mathbf{M C} \mathbf{C}^{(1)}$ \\
\hline & & & $\circ 2 \theta$ & $\mathrm{nm}$ \\
\hline \multirow[t]{2}{*}{$\mathrm{LV}_{1}$} & Test. & 0,81162 & 0,66889 & 11 \\
\hline & $\mathrm{C}_{3}$ & 0,73555 & 0,67079 & 12 \\
\hline \multirow[t]{2}{*}{$\mathrm{LV}_{2}$} & Test. & 0,58252 & 0,49734 & 21 \\
\hline & $\mathrm{C}_{3}$ & 0,73554 & 0,47451 & 18 \\
\hline \multirow[t]{2}{*}{$\mathrm{LVA}_{1}$} & Test. & 0,89091 & 0,64993 & 10 \\
\hline & $\mathrm{C}_{3}$ & 0,91734 & 0,67277 & 10 \\
\hline \multirow[t]{2}{*}{$\mathrm{LVA}_{2}$} & Test. & 0,82671 & 0,43033 & 18 \\
\hline & $\mathrm{C}_{3}$ & 0,74456 & 0,49169 & 17 \\
\hline \multirow[t]{2}{*}{$\mathrm{FX}$} & Test. & 0,33986 & 0,51565 & 38 \\
\hline & $\mathrm{C}_{3}$ & 0,65892 & 0,43524 & 22 \\
\hline
\end{tabular}

(1) Obtido de acordo com Keijser et al. (1983). Test.: testumenha; $\mathrm{C}_{3}$ : citrato $3 \mathrm{mmol} \mathrm{L}^{-1}$.

Quadro 4. Contrastes médios das médias das doses dos ácidos orgânicos dentro dos solos estudados para argila dispersa $(\mathrm{AD})$, resistência máxima à penetração $\left(\mathbf{R}_{\max }\right)$ e ferro $\left(\mathrm{Fe}_{\mathrm{o}}\right)$ e alumínio $\left(\mathrm{Al}_{\mathrm{o}}\right)$ extraídos por oxalato

\begin{tabular}{|c|c|c|c|c|c|c|c|c|}
\hline \multirow{2}{*}{ Solo } & \multicolumn{2}{|c|}{ AD } & \multicolumn{2}{|c|}{$\mathbf{R}_{\max }$} & \multicolumn{2}{|c|}{$\mathrm{Fe}_{\mathrm{o}}$} & \multicolumn{2}{|c|}{$\mathrm{Al}_{\mathrm{o}}$} \\
\hline & $\mathbf{C}_{1}$ & $\mathrm{C}_{2}$ & $\mathbf{C}_{1}$ & $\mathrm{C}_{2}$ & $\mathbf{C}_{1}$ & $\mathrm{C}_{2}$ & $\mathbf{C}_{1}$ & $\mathrm{C}_{2}$ \\
\hline $\mathrm{FX}$ & $51,18^{* *}$ & $6,15^{* *}$ & $4,91 * *$ & $-0,35^{\mathrm{ns}}$ & $0,06^{\mathrm{ns}}$ & $0,14^{\mathrm{ns}}$ & $-0,12^{\mathrm{ns}}$ & $-0,01^{\mathrm{ns}}$ \\
\hline $\mathrm{LVA}_{2}$ & $50,79^{* *}$ & $3,36^{\mathrm{ns}}$ & $11,50^{* *}$ & $7,45^{* *}$ & $0,01^{*}$ & $-0,07_{\mathrm{ns}}$ & $0,01^{\mathrm{ns}}$ & $-0,02^{* *}$ \\
\hline $\mathrm{LV}_{2}$ & $15,3^{* *}$ & $1,84^{\mathrm{ns}}$ & $4,05^{* *}$ & $-0,16^{\mathrm{ns}}$ & $-0,09^{\mathrm{ns}}$ & $-0,08^{*}$ & $-0,05^{\mathrm{ns}}$ & $-0,09^{\mathrm{ns}}$ \\
\hline $\mathrm{LV}_{1}$ & $67,6^{* *}$ & $9,84^{* *}$ & $3,57 * *$ & $1,09 * *$ & $0,1^{\mathrm{ns}}$ & $-0,01^{\mathrm{ns}}$ & $0,08^{\mathrm{ns}}$ & $0,01^{\mathrm{ns}}$ \\
\hline $\mathrm{LVA}_{1}$ & $42,88^{* *}$ & $1,21^{\mathrm{ns}}$ & $4,34^{* *}$ & $0,01^{\mathrm{ns}}$ & $0,08^{\mathrm{ns}}$ & $-0,08^{\mathrm{ns}}$ & $-0,03^{\mathrm{ns}}$ & $-0,01^{\mathrm{ns}}$ \\
\hline $\mathrm{RQ}$ & $34,00 * *$ & $2,22^{\mathrm{ns}}$ & $2,59 * *$ & $-0,04^{\mathrm{ns}}$ & $0,02^{\mathrm{ns}}$ & $0,01^{\mathrm{ns}}$ & $0,03^{\mathrm{ns}}$ & $0,01^{\mathrm{ns}}$ \\
\hline
\end{tabular}

$\mathrm{C}_{1}=\mathrm{AC}+\mathrm{AO}$. vs $\mathrm{AH}(++, 2-) ; \mathrm{C}_{2}=\mathrm{AC}$. vs AO. (+,-); AC: ácido cítrico; $\mathrm{AO}$ : ácido oxálico; $\mathrm{AH}$ : ácido húmico; *; **: significativos a 5 e $1 \%$; ns: não-significativo. 
ou atrativas vai depender da qualidade e teores de partículas minerais e complexantes orgânicos.

Ressalta-se que o trabalho considera somente efeitos de curto prazo (21 dias). Efeitos a longo prazo não devem ser desprezados, uma vez que o tempo é uma variável importante na formação e estabilização da estrutura do solo.

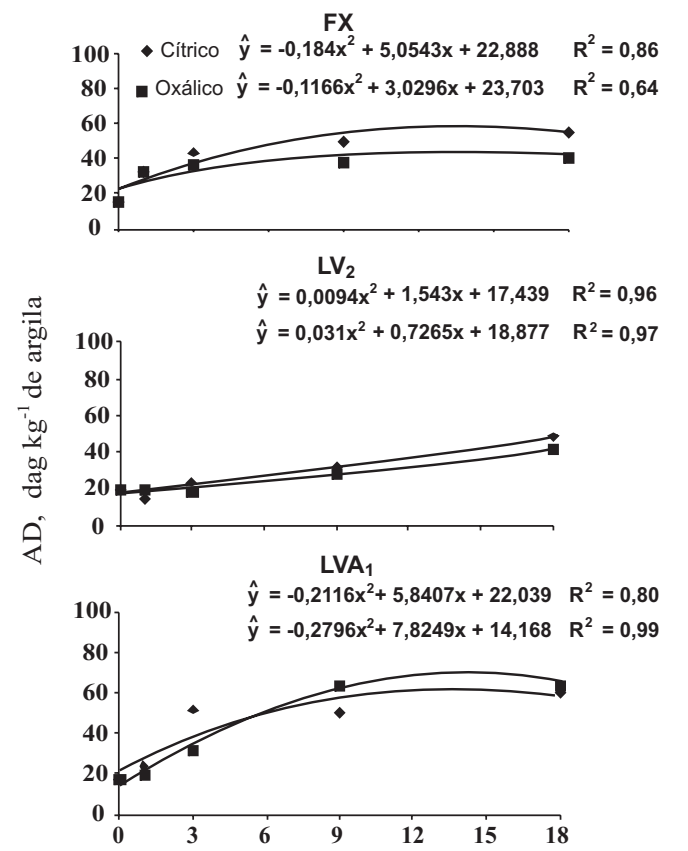

Além de variar com a dose dos ácidos, a $\mathrm{AD}$ também variou de acordo com a classe textural dos solos. Em geral, foram observados menores valores para solos de textura argilosa, conforme revelado pelo contraste $\mathrm{C}_{3}$ (Quadro 5). Esse fato deve-se, possivelmente, à grande presença de sítios de adsorção, em decorrência dos maiores teores de óxidos de Fe e Al no solo

DOSE, mmol L

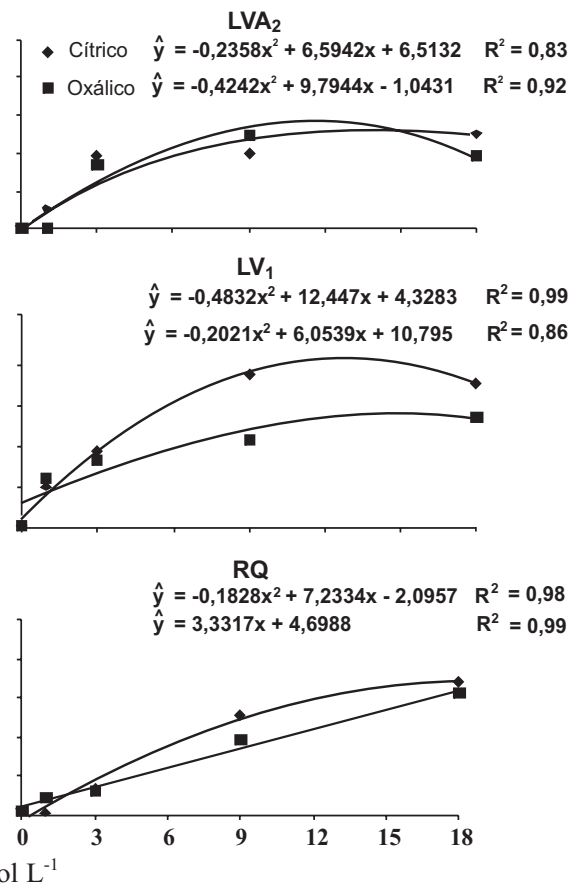

Figura 2. Efeito de doses dos ácidos cítrico e oxálico na argila dispersa em água (AD) para os diferentes solos estudados.

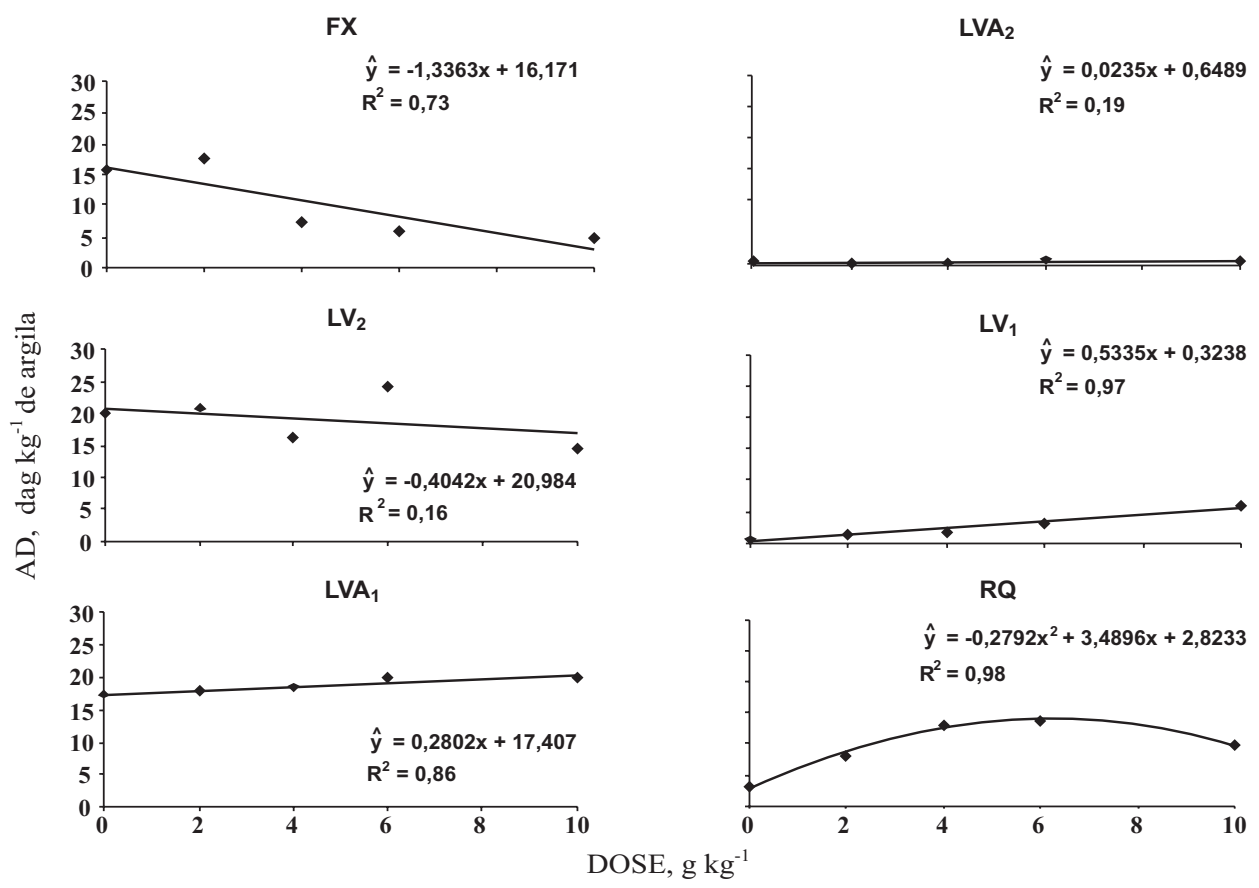

Figura 3. Efeito de doses de ácido húmico na argila dispersa em água (AD) para os diferentes solos estudados. 
Quadro 5. Contrastes médios para as médias de argila dispersa $(\mathrm{AD})$, resistência máxima à penetração $\left(R_{\max }\right)$ e ferro $\left(\mathrm{Fe}_{\mathrm{o}}\right)$ e alumínio $\left(\mathrm{Al}_{\mathrm{o}}\right)$ extraídos por oxalato dentro dos solos, de acordo com sua classe textural

\begin{tabular}{crrll}
\hline Contraste & AD & $\mathbf{R}_{\max }$ & $\mathbf{F} \mathbf{e}_{\mathbf{o}}$ & $\mathrm{A} \mathbf{l}_{\mathbf{o}}$ \\
\hline $\mathrm{C}_{3}$ & $-9,59^{* *}$ & $3,59^{* *}$ & $0,17^{* *}$ & $0,60^{* *}$ \\
$\mathrm{C}_{4}$ & $16,54^{* *}$ & $4,24^{* *}$ & $0,40^{* *}$ & $1,19^{* *}$ \\
$\mathrm{C}_{5}$ & $6,63^{* *}$ & $-2,29^{* *}$ & $0,28^{* *}$ & $0,22^{* *}$ \\
$\mathrm{C}_{6}$ & $9,67^{* *}$ & $0,75^{* *}$ & $-0,01^{\mathrm{ns}}$ & $0,14^{* *}$ \\
$\mathrm{C}_{7}$ & $-7,73^{* *}$ & $-1,89^{* *}$ & $0,22^{* *}$ & $0,43^{* *}$
\end{tabular}

$\mathrm{C}_{3}=\mathrm{LV}_{2}+\mathrm{LVA}_{2}+\mathrm{FX}$ vs $\mathrm{LVA}_{1}+\mathrm{LV}_{1}[+++, 1,5(-)] ; \mathrm{C}_{4=} \mathrm{LV}_{1}+$ $\mathrm{LVA}_{1}$ vs RQ (++, 2-); $\mathrm{C}_{5}=\mathrm{LV}_{2}$ vs $\mathrm{LVA}_{2}(+,-) ; \mathrm{C}_{6}=\mathrm{FX}$ vs LVA $(+,-) ; \mathrm{C}_{7}=\mathrm{LV}_{1}$ vs $\operatorname{LVA}_{1}(+,-) .{ }^{*} ; * *$ : significativos a 5 e $1 \%$; ns: não-significativo.

(Fernandes, 2000). Além disso, parte desses sítios encontra-se no interior de microagregados de alta estabilidade, típicos dos Latossolos (Schaefer, 2001), de difícil acesso para ação local dos ácidos.

Os valores de $\mathrm{AD}$ mostraram variação significativa entre os solos com mesma classe textural (Quadro 5), sugerindo a participação de outros fatores na resistência do solo à dispersão que não somente a adição dos ácidos orgânicos. Em geral, os valores de AD permitiram ordenar os solos, da seguinte forma: $\mathrm{LVA}_{2}<\mathrm{LV}_{2}<\mathrm{FX}<\mathrm{RQ}<\mathrm{LV}_{1}<\mathrm{LVA}_{1}$ (contrastes $\mathrm{C}_{5}$, $\mathrm{C}_{6}$ e $\mathrm{C}_{7}$, Quadro 5). Assim, percebe-se que, para a mesma classe textural, os solos goethíticos apresentaram maior resistência à dispersão que os hematíticos, e estes, por sua vez, maior que a dos gibbsíticos. Esse aspecto pode ser decorrente de variação na especificidade de adsorção dos ácidos pelos óxidos de $\mathrm{Fe}$ e $\mathrm{Al}$ (Varadachari et al., 2000). Esses resultados são corroborados por Tombácz et al. (2004), que verificaram variações na adsorção de ácidos orgânicos entre os óxidos, em que as hematitas apresentaram maior valor que as goethitas, maguemitas e gibbsitas. Os maiores valores de AD para o FX são provavelmente devidos à menor estabilidade de agregados no ambiente hidromórfico, sugerida pelo caráter plíntico desse solo.

\section{Ferro e alumínio extraídos por oxalato}

Não foi observada diferença significativa entre teores de $\mathrm{Fe}$ e $\mathrm{Al}$ extraídos por oxalato, quanto aos ácidos orgânicos estudados, como revelado pelos contrates $\mathrm{C}_{1}$ e $\mathrm{C}_{2}$ (Quadro 4). Esse fato sugere que, em curto prazo, tais ácidos não acarretaram dissolução desses elementos da estrutura cristalina e pobremente cristalizada de minerais dos solos. A não-percepção de alteração de cor dos solos para os diferentes tratamentos durante a condução do experimento também corrobora tal afirmação. A dominância dos óxidos de $\mathrm{Fe}$ e $\mathrm{Al}$ de melhor cristalinidade pode ser a principal razão dessa resistência ao fenômeno de dissolução pelos ácidos orgânicos.

\section{Resistência à penetração}

Os ácidos orgânicos de baixo peso molecular também apresentaram destacada influência na resistência à penetração (Figura 4). Em geral, quanto maior a dose aplicada, maiores foram os valores de máxima resistência $\left(R_{\max }\right)$.

Da mesma forma que $A D$, os valores de $R_{\text {max }}$ variaram de acordo com o ácido e se mostraram superiores para o ácido cítrico, principalmente no solo $\mathrm{LVA}_{2}$ (Figura 4). Nesse caso, o seu acentuado aumento $(0,21 \mathrm{kPa})$ não se relaciona com os valores de $\mathrm{AD}$, sugerindo a participação de outros fatores no adensamento desse solo. Conforme Marsan \& Torrent (1989), poder-se-ia esperar que esse aspecto fosse decorrente da forte interação entre materiais amorfos de $\mathrm{Si}$ com os óxidos de Fe, especialmente as goethitas. Esses autores encontraram alta relação entre os teores de $\mathrm{Si}_{\mathrm{d}}$ (extraído por DCB) e a dureza das porções amareladas de fragipãs da região mediterrânea.

Quanto ao solo $R Q$, o aumento da resistência à penetração com o aumento das doses do ácido cítrico foi devido à provável formação de pontes de ligação entre a matriz e o plasma argiloso disperso. Lamotte et al. $(1997 a, b)$ relataram que a dureza dos fragipãs de textura média a arenosa do nordeste de Camarões (África) foi conseqüência da presença dessas pontes, que integram fortemente as partículas grosseiras do solo. Esses autores estimaram um mínimo conteúdo de argila (6\%) para ocorrência desses materiais adensados, e essa propriedade (dureza) seria mais evidente com o aumento da proporção de argila fina (menor que $0,2 \mu \mathrm{m}$ ).

As figuras 4 e 5 indicam que o ganho na $R_{\max }$ do ácido húmico foi inferior ao dos ácidos cítrico e oxálico, corroborando os resultados obtidos por Shanmuganathan \& Oades (1983). Dessa forma, a ocorrência das camadas compactadas em áreas de plantio direto pode ser decorrente da liberação constante de ácidos de baixo peso molecular, como produto da decomposição dos resíduos orgânicos da superfície do solo. Os valores de $R_{\text {max }}$ praticamente não variam com o aumento da dose de ácido húmico, ocorrendo pequenas variações nos solos de textura média.

\section{Formação de horizontes adensados}

Os resultados sugerem que a resistência à penetração está altamente correlacionada com o teor de argila dispersa em água (Quadro 6, Figura 6), mostrando que esta pode ser uma importante variável para formação de camadas adensadas.

Por meio da relação entre $\mathrm{AD}$ e $\mathrm{R}_{\text {max }}$ (Figura 6), podem-se separar os solos estudados em dois grupos: aqueles que apresentaram pequeno incremento na resistência com grande aumento na $\mathrm{AD}\left(\mathrm{LV}_{1}, \mathrm{LVA}_{1} \mathrm{e}\right.$ 


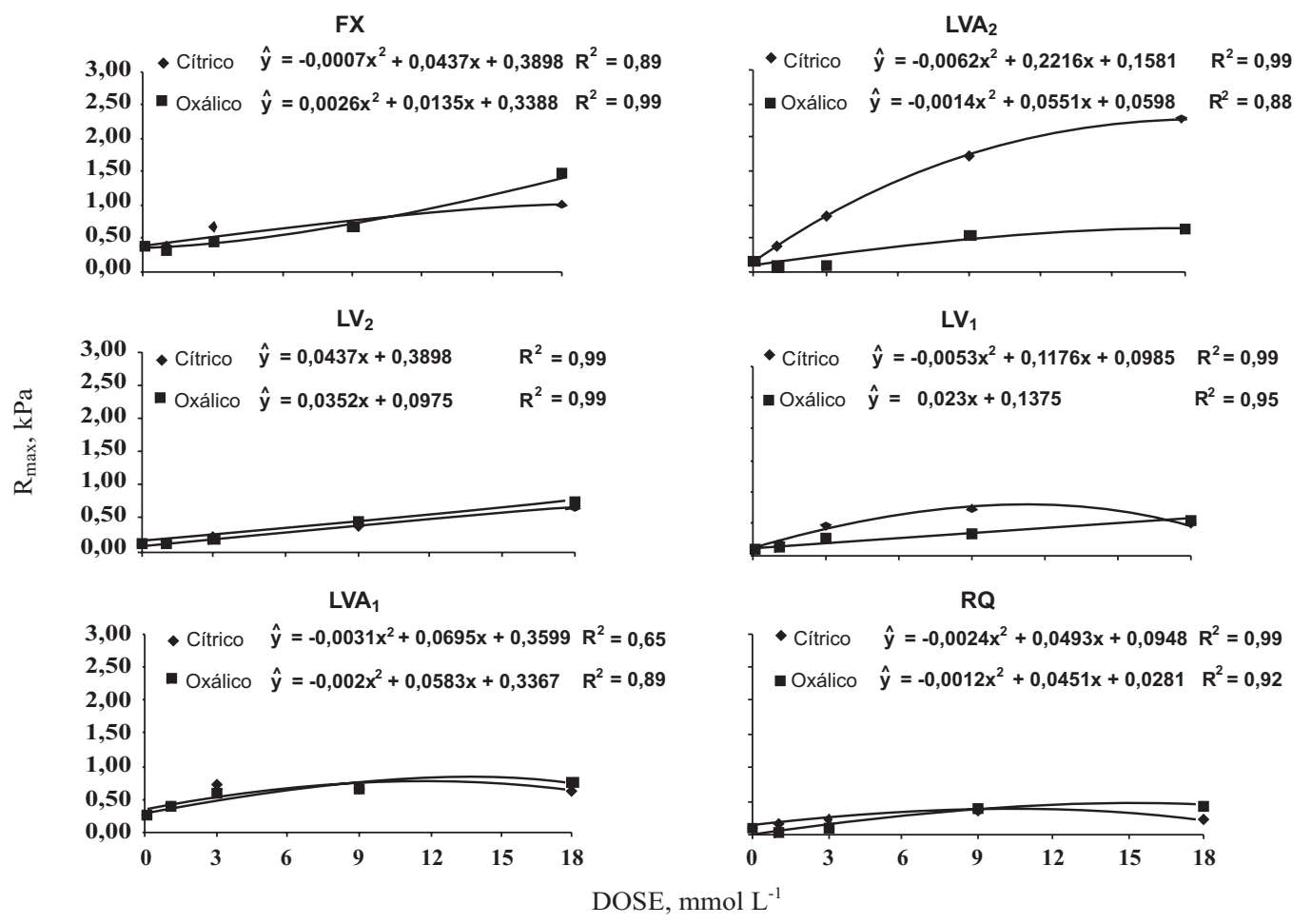

Figura 4. Efeito de doses dos ácidos cítrico e oxálico na resistência à penetração $\left(\mathbf{R}_{\max }\right)$ para os diferentes solos estudados.
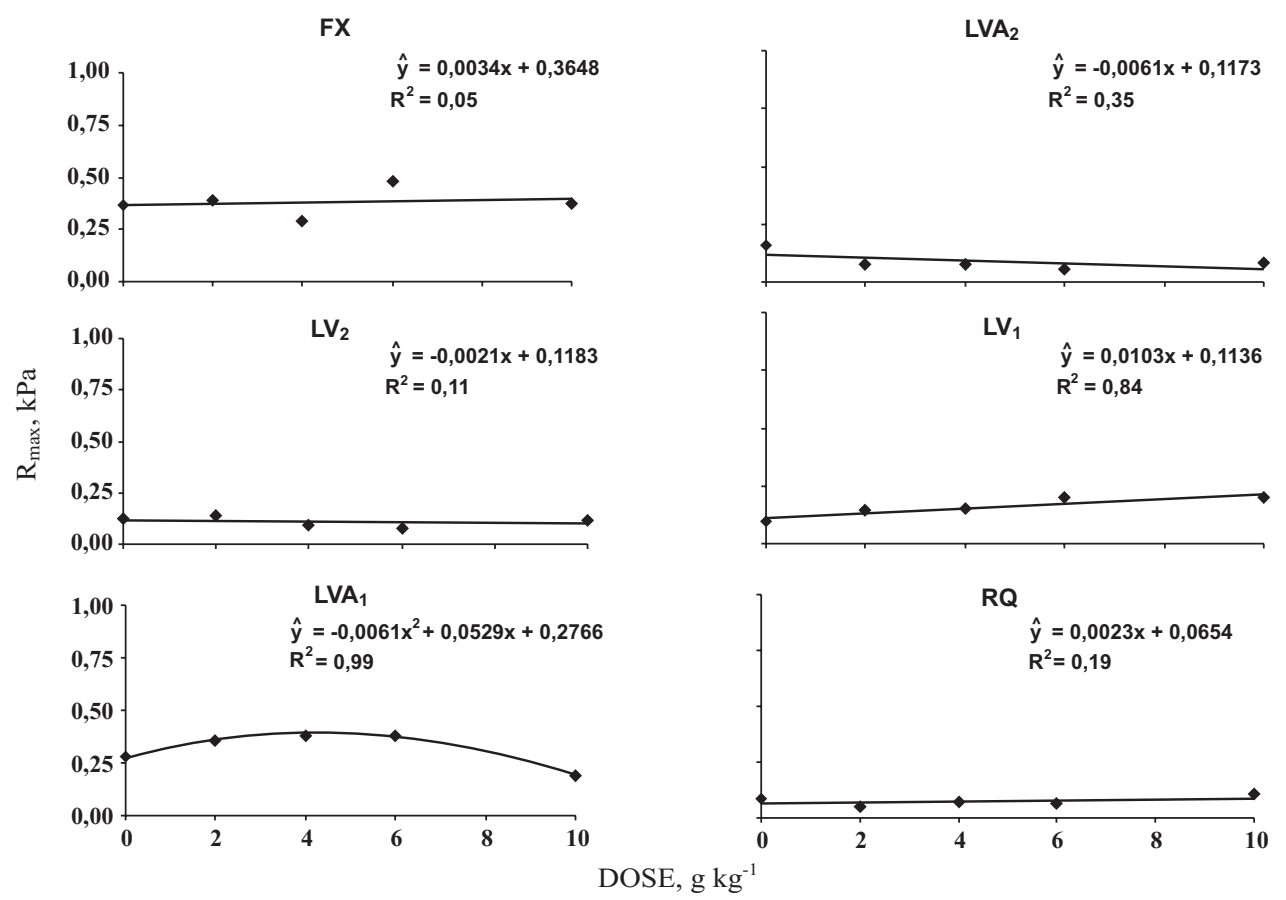

Figura 5. Efeito de doses de ácido húmico na resistência à penetração $\left(R_{\max }\right)$ para os diferentes solos estudados.

RQ), e aqueles em que um pequeno incremento em $\mathrm{AD}$ resultou em alto ganho na resistência $\left(\mathrm{FX}, \mathrm{LVA}_{2}\right.$ e $\mathrm{LV}_{2}$ ). Essa discretização condiz com o teor de argila desses solos. De forma geral, os solos argilosos (FX, $\mathrm{LVA}_{2}$ e $\mathrm{LV}_{2}$ ) apresentaram maior propensão ao adensamento que outros materiais. 

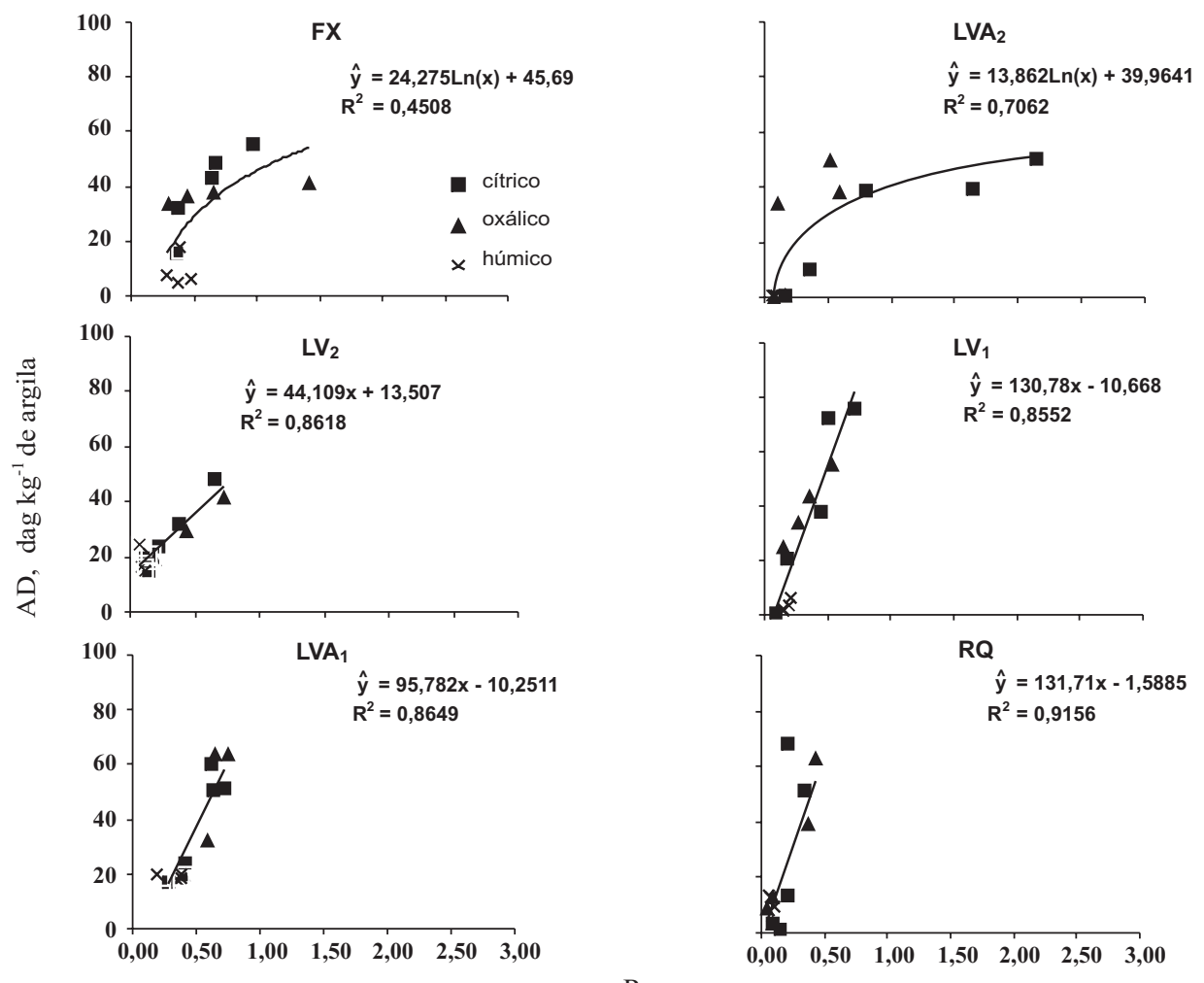

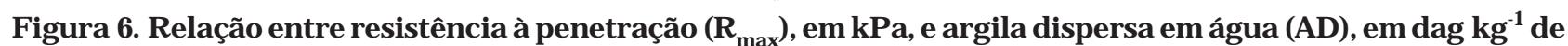
argila.

Não foi observada correlação significativa entre a $R_{\max }$ e os teores de $\mathrm{Fe}_{0}$ e $\mathrm{Al}_{0}$, com exceção do $\mathrm{LV}_{1}$, onde foi identificada tendência significativa a $20 \%$ com os valores de $\mathrm{Fe}_{0}$. A remoção desse elemento por complexação por meio da adição de ácidos orgânicos poderia diminuir a estabilidade dos agregados e, com isso, gerar altos teores de argila dispersa. Dessa forma, poderia ser explicado o alto incremento nos valores de $\mathrm{AD}$ para esse solo (Figura 2).

Quadro 6. Coeficientes de correlação entre a $\mathbf{R}_{\max } \mathbf{e}$ $\mathrm{AD}, \mathrm{Fe}_{\mathrm{o}}$ e $\mathrm{Al}_{\mathrm{o}}$ para os diferentes solos estudados

\begin{tabular}{|c|c|c|c|}
\hline \multirow{2}{*}{ Classe } & \multicolumn{3}{|c|}{ Coeficiente de correlação de $R_{\max }$ com: } \\
\hline & AD & Alo & Feo \\
\hline $\mathrm{FX}$ & $0,60^{*}$ & $-0,06 \mathrm{~ns}$ & $0,08^{\mathrm{ns}}$ \\
\hline LVA2 & $0,73^{* *}$ & $-0,09 \mathrm{~ns}$ & $-0,19 \mathrm{~ns}$ \\
\hline $\mathrm{LV} 2$ & $0,93 * * *$ & $-0,11 \mathrm{~ns}$ & $-0,16 \mathrm{~ns}$ \\
\hline LV1 & $0,92 * * *$ & $-0,13^{\mathrm{ns}}$ & $0,3820 \%$ \\
\hline LVA1 & $0,90 * * *$ & $0,03^{\mathrm{ns}}$ & $-0,13 \mathrm{~ns}$ \\
\hline $\mathrm{RQ}$ & $0,77 * *$ & $0,34^{\mathrm{ns}}$ & $0,12 \mathrm{~ns}$ \\
\hline
\end{tabular}

$\mathrm{R}_{\text {máx }}$ : resistência à penetração; $\mathrm{AD}$ : argila dispersa em água; $\mathrm{Fe}_{\mathrm{o}}$ : ferro extraído por oxalato ácido de amônio; $\mathrm{Al}_{\mathrm{o}}$ : alumínio extraído por oxalato ácido de amônio. $* * *, * *, *$ : significativos a $0,1,1$ e $5 \%$; ns: não-significativo.

\section{CONCLUSÕES}

1. O teor de argila dispersa variou com o tipo e dose dos ácidos orgânicos, textura e mineralogia dos solos, sugerindo que os solos goethíticos apresentaram maior resistência à dispersão que os hematíticos, e estes, maior que a dos gibbsíticos.

2. Os ácidos orgânicos de cadeia curta promoveram maiores dispersão de argila e resistência à penetração que os ácidos de cadeia longa, podendo-se estabelecer a seguinte ordem, de acordo com seu efeito: ácido cítrico $\geq$ ácido oxálico > ácidos húmicos.

3. O teor de argila dispersa em água é o principal fator no aumento da resistência do solo à penetração.

\section{LITERATURA CITADA}

ANDRADE, F.V. Ácidos orgânicos e adsorção de fosfato em Latossolos. Viçosa, MG, Universidade Federal de Viçosa, 2001. 42p. (Tese de Mestrado)

BENITES, V.M. \& MENDONCA, E.S. Propriedades eletroquímicas de um solo eletropositivo influenciadas pela adição de diferentes fontes de matéria orgânica. R. Bras. Ci. Solo, 22:215-221, 1998. 
CAGLiOTI, G.; PAOLETTI, A. \& RICCI, F.P. Choice of collimators for a crystal spectrometer for neutron diffraction. Nuclear Instr. Methods, 3:223-228, 1958.

DURGIN, P.B. \& CHANEY, J.G. Dispersion of kaolinite by dissolved organic matter from douglas-fir roots. Can. J. Soil Sci., 64:445-455, 1984.

EDWARDS, A.P. \& BREMNER, J.M. Microaggregates in soils. J. Soil Sci., 18:64-73, 1967.

EMERSON, W.W. Stability of soil crumbs. Nature, 183:538, 1959.

EMPRESA BRASILEIRA DE PESQUISA AGROPECUÁRIA EMBRAPA. Centro Nacional de Pesquisa de Solos. Manual de métodos de análise de solo. 2.ed. Rio de Janeiro, Ministério da Agricultura e do Abastecimento, 1997. 212p.

FERNANDES, R.B.A. Atributos mineralógicos, cor, adsorção e dessorção de fosfatos em Latossolos do Sudeste brasileiro. Viçosa, MG, Universidade Federal de Viçosa, 2000. 265p. (Tese de Doutorado)

GREENLAND, D.J. Interations between humic and fulvic acids and clays. Soil Sci., 111:34-41, 1971.

HANSBO, S. A New Approach to the Determination of the Shear Strength of Clay by the Fall-Cone Test. Proceeding of the 1957 Royal Suedish. Geotec. Inst., 14:7-47, 1957.

HINGSTON, F.J.; ATKINSON, R.J.; POSNER, A.M. \& QUIRK, L.P. Specific adsorption of anions. Nature, 215:1459-1461, 1967.

HUNTER, B.A. \& HOWARD, C.J. A computer program for Rietveld analysis of x-ray an neutron power diffraction patterns. Sydney, Australian Nuclear Science and Tecnology Organization, 2000. 27p.

KER, J.C. Mineralogia, sorção e dessorção de fosfatos, magnetização e elementos traços de Latossolos do Brasil. Viçosa, MG, Universidade Federal de Viçosa, 1995. 181p. (Tese de Doutorado)

LAMOTTE, M.; BRUAND, A.; HUMBEL, F.X.; HERBILLON, A. \& RIEU, M. A hard sandy-loam soil from semi-arid Northern Cameroon: I Fabric of the groundmass. Eur. J. Soil Sci., 48:213-225, 1997a.
LAMOTTE, M.; BRUAND, A.; OHNENSTETTER, D.; ILDEFONSE, P. \& PÉDRO, G. A hard sandy-loam soil from semi-arid Northern Cameroon: II Geochemistry and mineralogy of the bonding agent. Eur. J. Soil Sci., 48:227237, 1997b.

MACKEAGUE, J.A. Manual on soil sampling and methods of analysis. 2.ed. Ottawa, Canadian Society of Soil Science, 1978. 212p.

MARSAN, F.A. \& TORRENT, J. Fragipan bondind by silica and iron oxides in soil from northewest Italy. Soil. Sci. Am. J., 53:1140-1145, 1989.

SCHAEFER, C.E.G.R. The B horizon microstructure of brazilian Latosols as long-term biotic contructs. Aust. J. Soil Res., 39:909-926, 2001.

SHANMUGANATHAN, R.T. \& OADES, J.M. Influence of anions on dispersion and physical properties of the A horizon of a Red-Brown-Earth. Geoderma, 29:257-277, 1983.

SWIFT, R.S. Organic matter characterization. In: SPARKES, D. Methods of soil analysis, III Chemical methods. Soil Science Society of America Journal, Madison,1996. p.10181020 .

TARCHITZKY, J.; CHEN, Y. \& BANIN, A. Humic substances and $\mathrm{pH}$ effects on sodium and calcium-montmorillonite flocculation and dispersion. Soil Sci. Soc. Am. J., 57:367372, 1993.

TISDALL, J.M. \& OADES, J.M. Organic matter and waterstable aggregates in soils. J. Soil Sci., 62:141-163, 1982.

TOMBÁCZ, E.; LIBOR, Z.; ILLÉS, E.; MAJZIK, A. \& KLUMPP, E. The role of reactive surface sites and complexation by humic acids in the interaction of clay mineral and iron oxide particles. Org. Geogr., 35:257-267, 2004.

VARADACHARI, C.; CHATTOPADHYAY, T. \& GHOSH, K. The crystallo-quimistry of oxide-humus complexes. Aust. J. Soil Res., 38:789-806, 2000.

VISSER, S.A. \& CAILLIER, M. Observations on the dispersion and aggregation of clays by humic substances: I. Dispersive effects of humic acids. Geoderma, 42:331-337, 1988.

XU, R.; LI, C. \& JI, G. Effect of low-molecular-weight organic anions on electrokinetic properties of variable charge soils. J. Colloid Interface Sci., 277:243-247, 2004. 